\title{
Calabi-Yau threefolds with a curve of singularities and counterexamples to the Torelli problem
}

\author{
Balázs Szendrőin
}

3 June 1999

\begin{abstract}
Birational Calabi-Yau threefolds in the same deformation family provide a 'weak' counterexample to the global Torelli problem, as long as they are not isomorphic. In this paper, it is shown that deformations of certain desingularized weighted projective hypersurfaces provide examples of families containing birational varieties. The constructed examples are shown to be non-isomorphic using a specialization argument.
\end{abstract}

AMS Subject Classification: 14J32, 14C34

\section{Introduction}

The theory of Calabi-Yau threefolds has received a lot of attention in recent years, due to its relation to various fields ranging from birational geometry to superstring theory. One of the important problems in the theory is the

Torelli Question: Let $Y_{1}, Y_{2}$ be smooth, deformation equivalent Calabi-Yau threefolds over $\mathbb{C}$. Assume that there is an isomorphism

$$
\left(H^{3}\left(Y_{1}\right), Q_{Y_{1}}\right) \cong\left(H^{3}\left(Y_{2}\right), Q_{Y_{2}}\right)
$$

respecting the Hodge structures polarized by the intersection forms. Are $Y_{1}$ and $Y_{2}$ isomorphic?

The answer to this question remained elusive for a long time even in the simplest case, for general quintic hypersurfaces in $\mathbb{P}^{4}$. However, weak global Torelli in this case has recently been proved by Voisin [19]. It is possible that the answer is positive for simply connected Calabi-Yau threefolds with Picard number one.

In this paper, I give some examples of families of threefolds where the answer to the above question is negative. They are in a sense 'weak' examples. They arise from a theorem of Kollár, which claims that there is an isomorphism of polarized Hodge structures as above if $Y_{1}, Y_{2}$ are birational Calabi-Yau threefolds. In Section 1, I exhibit birational threefolds which are at

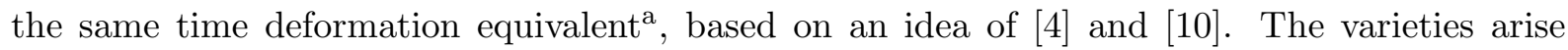
as deformations of resolutions $Y$ of Calabi-Yau threefolds with a curve of singularities. The birational isomorphisms give an interesting involution on the base of the Kuranishi space of the resolutions $Y$.

In order to obtain a counterexample to Torelli, one has to prove that the constructed smooth threefolds are not isomorphic. I will introduce three families of explicit examples in Section 2 that exhibit three different sorts of behaviour. The difference lies in the generic automorphism group and the action of this group on the Kuranishi space. In particular, Proposition 2.4 shows that in one family the presence of an involution destroys the counterexample. The main result

\footnotetext{
${ }^{\dagger}$ Supported by an Eastern European Research Bursary from Trinity College, Cambridge and an ORS Award from the British Government.

${ }^{\text {a}}$ For this phenomenon to occur, the Picard number must be greater than one, as in the Picard number one case the smooth projective birational model is unique.
} 
of the paper is Theorem 4.4, which gives explicit cases where (weak) global Torelli fails. In particular, a question posed in [3, 1.6] is answered. The proof is based on a specialization argument and a standard result on automorphisms in families.

The construction shows that one can find threefolds with the same Hodge structure in an arbitrary small disc neighbourhood of the central fibre in the Kuranishi space of $Y$. This however does not contradict Infinitesimal Torelli: as explained in Remark 4.5, the period point of $Y$ in the period domain is fixed by an element of the arithmetic monodromy group.

Desingularizations of Horrocks-Mumford quintics give another example of birational, deformation equivalent Calabi-Yau threefolds [2], the Picard number being four in that case. Indeed, it is reasonable to expect that as the Picard number increases, Torelli can fail more and more badly, although under a weak condition there will always be finitely many isomorphism classes with the same Hodge structure within a deformation family by the main result of [15].

The examples suggest the

Modified Torelli Question: Let $Y_{1}, Y_{2}$ be smooth, deformation equivalent Calabi-Yau threefolds over $\mathbb{C}$, with isomorphic polarized Hodge structures. Are $Y_{1}$ and $Y_{2}$ birationally equivalent?

The example of Aspinwall-Morrison [1], featuring non-simply connected threefolds, may provide a counterexample to this more general question, but some details of that example remain to be worked out

My final remark is that the theory of holomorphic symplectic varieties shows somewhat parallel features. On one hand, there is an example of birational, non-isomorphic varieties [6]. On the other hand, the polarized Hodge structures (on second cohomology) of birational varieties are isomorphic, and the natural way to pose the Torelli problem is exactly the same as above [9]. However, similarities end here, [9, 4.6] shows that two birational holomorphic symplectic manifolds can always be realized as central fibres in families over the disc, the families being isomorphic over the punctured disc. This phenomenon is special to the holomorphic symplectic case.

Acknowledgements I wish to thank Pelham Wilson for suggesting the idea of the construction, and also for encouragement, comments and corrections. Thanks are also due to Miles Reid for his interest in my work and friendly advice, to Michael Fryers, Daniel Huybrechts, Umar Salam and Burt Totaro for discussions and comments and finally to the referee for suggesting some improvements and pointing out Proposition 4.6.

\section{Notation and definitions}

A Calabi-Yau threefold is a normal projective threefold $Y$ with canonical Gorenstein singularities, satisfying $K_{Y} \sim 0$ and $H^{1}\left(Y, \mathcal{O}_{Y}\right)=0$. The nef cone of $Y$ is the closed cone generated by ample classes in $\operatorname{Pic}(Y) \otimes \mathbb{R} \cong H^{2}(Y, \mathbb{R})$.

The weighted projective space $\mathbb{P}^{m}\left[w_{0}, \ldots w_{m}\right]$ is the quotient of $\mathbb{C}^{n+1} \backslash\{0\}$ by the $\mathbb{C}^{*}$-action having the given weights. A weighted hypersurface $X=X_{d} \subset \mathbb{P}^{m}\left[w_{0}, \ldots w_{m}\right]$ is defined by the vanishing of a homogeneous polynomial $f$ of weighted degree $d$.

If $Y$ is a variety, by a slight abuse of notation I write $T_{0} \operatorname{Def}(Y)$ for the linear space classifying first order deformations of $Y$. This space is isomorphic to $H^{1}\left(Y, \Theta_{Y}\right)$. If $f: Y \rightarrow X$ is a

\footnotetext{
${ }^{\mathrm{b}} \mathrm{A}$ detailed analysis of the example will be given in 16 .
} 
morphism of algebraic varieties, a deformation of $f$ over $S$ is a commutative diagram

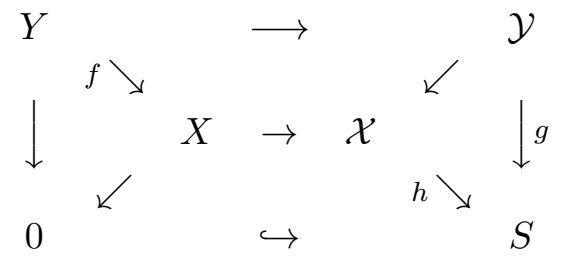

where $g, h$ are flat and $Y=g^{-1}(0), X=h^{-1}(0)$. There is a vector space $T_{0} \operatorname{Def}(Y, f, X)$ classifying the first order deformations, defined defined by Ran [14] as a complicated Ext group over a Grothendieck topology. There are natural maps

$$
T_{0} \operatorname{Def}(X) \leftarrow T_{0} \operatorname{Def}(Y, f, X) \rightarrow T_{0} \operatorname{Def}(Y)
$$

to the first order deformation spaces of $X$ and $Y$.

Finally, $H^{n}(X)$ denotes the integral cohomology of $X$ modulo torsion.

\section{The general framework}

The counterexamples to the Torelli problem presented in this paper will be based on the following result:

Theorem 1.1 (Kollár [11, 4.12-13].) Assume that $Y_{1}, Y_{2}$ are smooth Calabi-Yau threefolds, related by a flop $\psi: Y_{1} \rightarrow Y_{2}$. Then $\psi$ induces an isomorphism of polarized Hodge structures

$$
\left(H^{3}\left(Y_{1}\right), Q_{Y_{1}}\right) \cong\left(H^{3}\left(Y_{2}\right), Q_{Y_{2}}\right) \text {. }
$$

As a first step, I introduce a class of Calabi-Yau threefolds having birational varieties in their deformation space.

Construction 1.2 Let $Y$ be a smooth Calabi-Yau threefold containing a surface $E$ ruled over a curve $C$ of genus $g \geq 2$, necessarily smooth by [20], [21], which can be contracted inside $Y$ by a log-extremal contraction given by the divisor $H \in \operatorname{Pic}(Y)$ :

$$
\begin{aligned}
\varphi_{|m H|}: & Y \rightarrow X \\
& \rightarrow \\
E & \rightarrow C .
\end{aligned}
$$

For the rest of the paper, let $\pi: \mathcal{Y} \rightarrow S$ be the the Kuranishi family of $Y$. By Unobstructedness [17], [18] its base $S$ is smooth. Fix once and for all an identification between $S$ and an open disc in $T_{0} \operatorname{Def}(Y)$; this defines a notion of (partial) addition and scalar multiplication on $S$. There is an obvious one-to-one correspondence between vectors $v \in S$ and maps from the unit disc $f_{v}: \Delta \rightarrow S$, given by $f_{v}(z)=z v$. The map $f_{v}$ gives a pullback family $\pi_{v}: \mathcal{Y}_{v} \rightarrow \Delta$ with smooth Calabi-Yau fibres.

Let

$$
S_{E}=\operatorname{Im}\left(T_{0} \operatorname{Def}(E, k, Y) \rightarrow T_{0} \operatorname{Def}(Y)\right) \cap S,
$$

this is a linear subspace of $S$, corresponding to the deformation directions along which $E$ deforms together with the deformation. Wilson [20] shows that if $E$ is smooth, $S_{E} \subset S$ is of codimension $g$.

Let $\pi_{v}: \mathcal{Y}_{v} \rightarrow \Delta$ be a general one-parameter deformation for $v$ not contained in $S_{E}$. Assume that the nef cone is invariant in the family. (This will be proved for all the discussed examples in Lemma 2.7.) The following is well-known: 
Proposition 1.3 There exists a unique relative Cartier divisor $\mathcal{H}$ on $\mathcal{Y}_{v}$ extending $H$ on $Y$. There is a morphism $\varphi_{|m \mathcal{H}|}: \mathcal{Y}_{v} \rightarrow \mathcal{X}_{v}$ over $\Delta$, which is the contraction of $E$ in the central fibre and the contraction of a finite number of rational curves $\cup_{i} C_{t}^{i}$ on the general fibre $Y_{t}$, the number of such curves being $2 g-2$ if counted with appropriate multiplicities. The map $\mathcal{X}_{v} \rightarrow \Delta$ is flat.

Proof As $h^{2}\left(\mathcal{O}_{Y}\right)=0$, the divisor $H$ extends uniquely over the family, and by assumption $\mathcal{H}_{t}$ is a nef and big divisor on all fibres $Y_{t}$. So $\pi_{v *}(m \mathcal{H})$ is a vector bundle over $\Delta$ and $|m \mathcal{H}|$ defines a morphism. The exceptional locus over $t \in \Delta^{*}$ is a finite union of rational curves by [20], their expected number (Gromov-Witten invariant) is calculated in [22, 2.3]. The last statement follows from [13, 11.4].

Proposition 1.4 (cf. [22, 2.3].) There exists a flop of $\mathcal{Y}_{v} \rightarrow \Delta$, i.e. a diagram

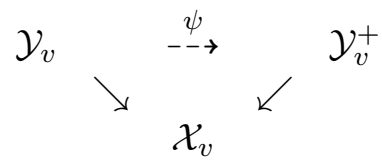

over $\Delta$, where the birational map $\psi_{t}$ flops the curves $C_{t}^{i}$ on $Y_{t}$ for $t \in \Delta^{*}$ and gives an isomorphism on the fibre over 0 .

Proof $\mathcal{X}_{v}$ has only cDV singularities, so the existence of the flop can be seen by taking hyperplane sections and using [13, 11.10]. $\mathcal{Y}_{v}^{+}$is smooth, so the birational map $\mathcal{Y}_{v} \rightarrow \mathcal{Y}_{v}^{+}$is an isomorphism in codimension 1 , which shows that $\psi_{0}$ is an isomorphism.

The family $\mathcal{Y}_{v}^{+} \rightarrow \Delta$ has central fibre $Y$, so corresponds to some vector $\alpha(v) \in H^{1}\left(Y, T_{Y}\right)$. Shrinking $S$ if necessary and defining $\alpha$ as the identity on $S_{E}$, one obtains a map $\alpha: S \rightarrow S$ which is clearly an involution. The following is a tautology:

Proposition 1.5 The map $\alpha$ is linear in the obvious sense. Its fixed locus is exactly $S_{E}$.

Proof Let $v_{1}, v_{2} \in S \backslash S_{E}$ such that $v=v_{1}+v_{2} \in S$ also, then the maps $f_{v_{i}}$ together with the linear structure on $S$ give a map $\Delta^{2} \rightarrow S$. The map $f_{v}$ coincides with the composite map $\Delta \rightarrow \Delta^{2} \rightarrow S$ where the first map is the diagonal one. The flop can just as well be constructed over $\Delta^{2}$ which shows linearity immediately. The obvious modification of this argument works also if some $v_{i} \in S_{E}$.

Now suppose $v$ is not in $S_{E}$ but it is fixed by $\alpha$. By universality of the Kuranishi family, this means that the birational map $\psi$ is the identity on all fibres. This is clearly nonsense.

\section{Particular families}

In this Section, I will briefly investigate the geometry of three families which fit into the framework described above. The singular variety $X$ will be a general hypersurface $X_{8} \subset \mathbb{P}^{4}\left[1^{2}, 2^{3}\right]$, $X_{12} \subset \mathbb{P}^{4}\left[1^{2}, 2^{2}, 6\right]$ or $X_{14} \subset \mathbb{P}^{4}\left[1,2^{3}, 7\right]$, respectively. $X$ has Picard number one, canonical singularities along curves and by adjunction, trivial canonical sheaf.

2.1 First assume that $X=X_{8} \subset \mathbb{P}^{4}\left[1^{2}, 2^{3}\right]$, a variety discussed at length from the point of view of mirror symmetry in [⿶], deformations of which also featured in [3, 1.6]. $X$ is singular along the locus $C=\left\{x_{i}=0\right\}$, a plane curve of genus 3 . 
The linear system $\mathcal{O}(2)$ embeds $\mathbb{P}^{4}\left[1^{2}, 2^{3}\right]$ as a quadric $Q_{3}=\left\{z_{1} z_{3}=z_{2}^{2}\right\}$ of rank 3 in $\mathbb{P}^{5}$, where $z_{i}$ are the coordinates on the $\mathbb{P}^{5}$. The hypersurface $X$ becomes a complete intersection of $Q_{3}$ with a quartic $F_{4}$ in $\mathbb{P}^{5}$. The singularities of the quadric $Q_{3}$ can be resolved by the map $F(2,0,0,0) \rightarrow Q_{3}$, where $F(2,0,0,0)$ is a rational scroll. $F(2,0,0,0)$ generically deforms to the scroll $F(1,1,0,0)$. The contraction $\varphi: F(1,1,0,0) \rightarrow Q_{4} \subset \mathbb{P}^{5}$ maps the scroll to a quadric $Q_{4}=\left\{z_{1} z_{3}=z_{2}^{2}-t z_{4}^{2}\right\}$ of rank 4 . Intersections of $Q_{4}$ with $F_{4}$ give deformations $X_{t}$ of $X$, with four isolated $c A_{1}$ points at $\left\{z_{1}=\ldots=z_{4}=0\right\}$. The resolution $Y_{t} \rightarrow X_{t}$ replaces these points by the rational curves $C_{t}^{i}$. The following diagram summarizes the state of affairs:

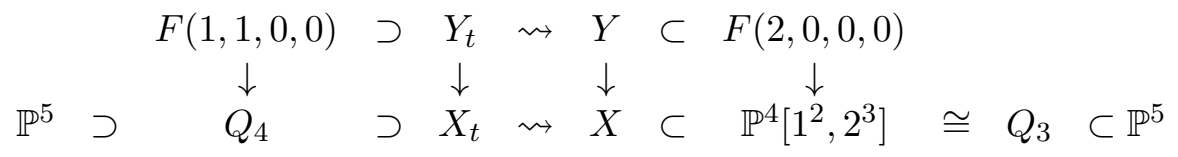

$F(1,1,0,0)$ is a quotient of $\left(\mathbb{C}^{2} \backslash\{0\}\right) \times\left(\mathbb{C}^{4} \backslash\{0\}\right)$ by the group $\left(\mathbb{C}^{*}\right)^{2}$, where the two multiplicative actions have weights $(1,1 ;-1,-1,0,0)$ and $(0,0 ; 1,1,1,1)$ respectively. If $t_{i}, u_{i}$ denote the coordinates on the affine spaces, then the map $\varphi$ is given by

$$
\varphi:\left(t_{1}, t_{2} ; u_{1}, \ldots, u_{4}\right) \mapsto\left(u_{1} t_{1}: \frac{1}{2}\left(u_{1} t_{2}+u_{2} t_{1}\right): u_{2} t_{2}: \frac{1}{2 \sqrt{t}}\left(u_{1} t_{2}-u_{2} t_{1}\right): u_{3}: u_{4}\right),
$$

having the quadric $Q_{4}$ as its image. There is however an ambiguity in the choice of the sign of the square root. This does not matter in $\mathbb{P}^{5}$, as the two choices are isomorphic under the map $\sigma: \mathbb{P}^{5} \rightarrow \mathbb{P}^{5}, z_{4} \mapsto-z_{4}, z_{1} \leftrightarrow z_{3}$. However, taking the intersection with the quartic $F_{4}$, the two choices give different resolutions as long as $\sigma$ is not an automorphism of $F_{4}$. This gives the two resolutions related by a flop.

Note that if $\sigma$ is an automorphism of $F_{4}$, then it gives an automorphism $j \in \operatorname{Aut}(Y)$ and the map $\alpha$ acting on the base of the Kuranishi space $S$ of $Y$ equals $j^{*}$. For such special points in the moduli space, the families $\mathcal{Y}_{v}, \mathcal{Y}_{\alpha(v)}$ are isomorphic.

2.2 Next consider the other two families $X_{12} \subset \mathbb{P}^{4}\left[1^{2}, 2^{2}, 6\right]$ and $X_{14} \subset \mathbb{P}^{4}\left[1,2^{3}, 7\right]$. These varieties are singular along the curves given by the vanishing of the variables of odd degrees, of genus 2, 15 respectively.

Notice that these varieties always have nontrivial automorphisms. If $z$ denotes the variable of highest degree and $x_{i}, y_{i}$ the other variables, then changing variables $X=\left\{z^{2}+f_{d}\left(x_{i}, y_{i}\right)=0\right\}$ and then $z \mapsto-z$ gives an involution $i$. The automorphism $i$ of $X$ extends to an involution $j$ on the resolution $Y . j$ induces a natural linear action $j^{*}$ on the space $T_{0} \operatorname{Def}(Y)$; denote its fixed locus by $T_{0}^{+} \operatorname{Def}(Y)$. This is the subspace of $T_{0} \operatorname{Def}(Y)$ corresponding to deformation directions along which the involution $j$ also deforms. Notice that by universality of the Kuranishi family, the families $\mathcal{Y}_{v} \rightarrow \Delta, \mathcal{Y}_{j^{*}(v)} \rightarrow \Delta$ are isomorphic under an isomorphism induced by $j$. The following is again a tautology:

Proposition 2.3 The actions of $\alpha, j^{*}$ on $S$ commute.

Proof The following diagram obviously commutes:

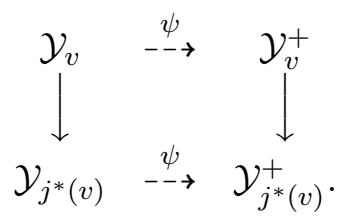

Thus $\mathcal{Y}_{j^{*} \alpha(v)}=\mathcal{Y}_{j^{*}(v)}^{+}=\mathcal{Y}_{\alpha j^{*}(v)}$.

In the two cases, the involution $j^{*}$ behaves very differently: 
Proposition 2.4 If $Y$ is the resolution of $X_{12} \subset \mathbb{P}^{4}\left[1^{2}, 2^{2}, 6\right]$, then $j^{*}$ is trivial. If $Y$ is the resolution of $X_{14} \subset \mathbb{P}^{4}\left[1,2^{3}, 7\right]$, then $j^{*}=\alpha$ as maps acting on the base of the Kuranishi space of $Y$.

Proof Let $X=X_{12} \subset \mathbb{P}^{4}\left[1^{2}, 2^{2}, 6\right]$, a double cover $X \rightarrow \mathbb{P}^{3}\left[1^{2}, 2^{2}\right] \cong Q_{3} \subset \mathbb{P}^{4}$ of a quadric of rank 3 in $\mathbb{P}^{4}$, branched over a sextic. The quadric is resolved by the scroll $F(2,0,0) \rightarrow Q_{3}$, and $Y$ is a branched double cover of this scroll. The variety $Y$ appears in a paper of Fujita [8], where it is denoted by $\Sigma^{3}(2,0,0)_{3,0}^{+}$. By [8, 7.13], the action of $j^{*}$ on $S$ is trivial. This proves the first statement.

Now let $X=X_{14} \subset \mathbb{P}^{4}\left[1,2^{3}, 7\right]$. The fixed locus of the involution $i: z \rightarrow(-z)$ is in this case reducible; the quotient is $X /\langle i\rangle \cong \mathbb{P}^{3}\left[1,2^{3}\right] \cong \mathbb{P}^{3}$. The image of the curve $C$ is $\left\{t_{1}=0\right\} \cap\left\{g_{7}\left(t_{1}, \ldots t_{4}\right)=0\right\}=\gamma$. The involution extends to the resolution $Y$ as an involution $j$. The quotient upstairs is $Y /\langle j\rangle \cong W=B_{\gamma} \mathbb{P}^{3}$, the blowup of $\mathbb{P}^{3}$ along the curve $\gamma$. One obtains a diagram

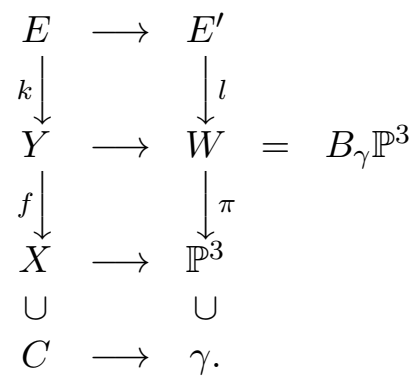

Let $\Theta_{W}$ be the tangent bundle of $W, N_{\gamma / \mathbb{P}^{3}}$ the normal bundle of $\gamma$ in its ambient space. Standard arguments show

Lemma 2.5 The natural map $H^{0}\left(\gamma, N_{\gamma / \mathbb{P}^{3}}\right) \rightarrow H^{1}\left(W, \Theta_{W}\right)$ is surjective, i.e. any (first-order) deformation of $W$ comes from a deformation of $\gamma$ in $\mathbb{P}^{3}$.

Lemma 2.6 There is an inclusion

$$
T_{0}^{+} \operatorname{Def}(Y) \subset \operatorname{Im}\left(T_{0} \operatorname{Def}(E, k, Y) \rightarrow T_{0} \operatorname{Def}(Y)\right) .
$$

Proof Consider

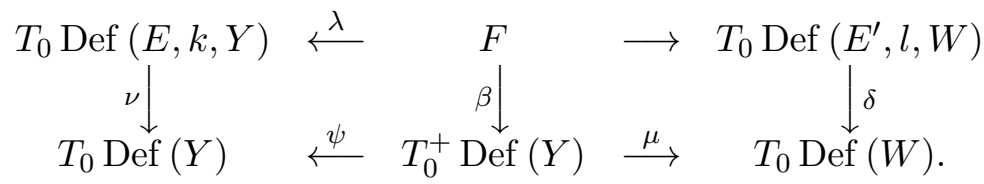

Here $\mu, \delta, \nu$ are the obvious maps and $\psi$ is the inclusion. Let $F$ be the fibre product; its elements are pairs of vectors giving a first-order deformation $\mathcal{Y} \rightarrow B=\operatorname{Spec} \mathbb{C}[\epsilon] /\left(\epsilon^{2}\right)$ of $Y$ with an involution $J$ on $\mathcal{Y}$, and a first-order deformation $\left(\mathcal{W}, \mathcal{E}^{\prime}\right) \rightarrow B$ of the inclusion $l: E^{\prime} \rightarrow W$, with compatible image in $T_{0} \operatorname{Def}(W)$. In other words, elements of $F$ give a diagram

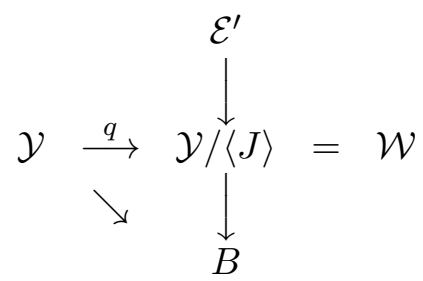


where $q$ is the quotient map and all maps over $B$ are flat. $q$ is finite, so $\mathcal{E}=q^{-1}\left(\mathcal{E}^{\prime}\right)$ is a relative Cartier divisor on $Y$, flat over $B$, hence one obtains a deformation $\mathcal{E} \rightarrow \mathcal{Y}$ of $E \rightarrow Y$. This defines the map $\lambda$ in the previous diagram. By construction, the left square becomes commutative.

Lemma 2.5 shows that $\delta$ is surjective, so $\beta$ must also be surjective. Then the commutativity of the left square shows that the image of the inclusion $\psi$ must be contained in the image of $\nu$. This proves Lemma 2.6.

To complete the proof of Proposition 2.4, notice that $j^{*}=\alpha$ would follow from the equality of fixed loci, as the two maps are commuting involutions, linear with respect to the partial linear structure on $S$. The fixed locus of $\alpha$ is $S_{E}$, whereas the fixed locus of $j^{*}$ is by definition $T_{0}^{+} \operatorname{Def}(Y) \cap S$. By the previous Lemma, $S_{E} \supset T_{0}^{+} \operatorname{Def}(Y) \cap S$ and it suffices to show that the dimensions here are equal. The dimension of $S_{E}$ is $h^{2,1}(Y)-g(C)=107$. On the other hand, by a standard dimension count, the hypersurface $X_{14} \subset \mathbb{P}^{4}\left[1,2^{3}, 7\right]$ depends on 107 parameters. Resolutions of such hypersurfaces are always double covers, so $\operatorname{dim} T_{0}^{+} \operatorname{Def}(Y) \geq 107$. This concludes the proof.

Thus in the last case, the varieties $Y_{t}, Y_{\alpha(t)}$ are isomorphic for all $t$. For the rest of the paper, restrict attention to the first two families. The last statement in this Section describes the nef cone of $Y$ in these cases; this description will be needed below.

Proposition 2.7 The nef cone of the resolution $Y$ is generated by the flopping face and the face corresponding to a fibration with base $\mathbb{P}^{1}$. It is constant on any deformation of $Y$.

Proof The first statement is clear from the above discussion; the map to $\mathbb{P}^{1}$ comes from the structural map of the rational scrolls. As for the second part, by the main result of [20], the nef cone is invariant on all deformations if no deformation of $Y$ contains a surface which is (quasi-) ruled over an elliptic curve. A sufficient condition for this is that there is no class $F \in H^{2}(Y, \mathbb{Q})$ satisfying $F^{3}=c_{2}(Y) \cdot F=0 . H, E \in H^{2}(Y, \mathbb{Q})$ are not linearly dependent, so they give a $\mathbb{Q}$-basis of the rank-two space $H^{2}(Y, \mathbb{Q})$ and the existence of the class $F$ is equivalent to $\left(\left(c_{2}(Y) \cdot H\right) E-\left(c_{2}(Y) \cdot E\right) H\right)^{3}=0$. A routine calculation shows that this fails in both cases.

Remark 2.8 The families investigated in this Section belong to a larger set of examples that can be found using a systematic search based on the theory of graded rings of weighted complete intersection varieties. For details, consult [16].

\section{The automorphism group of the general variety}

Proposition 3.1 The general $X_{8} \subset \mathbb{P}^{4}\left[1^{2}, 2^{3}\right]$ has trivial automorphism group, whereas the general $X_{12} \subset \mathbb{P}^{4}\left[1^{2}, 2^{2}, 6\right]$ has automorphism group $\mathbb{Z} / 2 \mathbb{Z}$.

Proof Assume that $\sigma$ is a nontrivial element of the automorphism group of the hypersurface $X=\left\{F_{8}(\vec{x}, \vec{y})=0\right\} \subset \mathbb{P}^{4}\left[1^{2}, 2^{3}\right]$,

$$
F_{8}(\vec{x}, \vec{y})=f_{8}(\vec{x})+g_{1}(\vec{y}) f_{6}(\vec{x})+g_{2}(\vec{y}) f_{4}(\vec{x})+g_{3}(\vec{y})\left(x_{1}^{2}+x_{2}^{2}\right)+g_{4}(\vec{y}),
$$

where $\vec{x}=\left(x_{j}\right), \vec{y}=\left(y_{j}\right)$ are the homogeneous coordinates of degrees 1,2 respectively. The Picard group of $X$ is of rank one, which implies that $\sigma$ comes from a projective automorphism 
of $\mathbb{P}^{4}\left[1^{2}, 2^{3}\right]$. Hence by [5, 4.7$]$,

$$
\begin{aligned}
\sigma(\vec{x}) & =A \vec{x}, \\
\sigma(\vec{y}) & =B \vec{y}+C\left(S^{2} \vec{x}\right),
\end{aligned}
$$

where $A$ is a $2 \times 2$ matrix, $B, C$ are $3 \times 3$ matrices and $S^{2} \vec{x}$ is a shorthand for $\left(x_{1}^{2}, x_{1} x_{2}, x_{2}^{2}\right)^{t}$.

The singular locus of $X$ is the genus three plane curve $\left\{g_{4}=0\right\} \subset \mathbb{P}^{2}$. This has to be mapped isomorphically by $\sigma$, so if $g_{4}$ is general, then after fixing an overall constant, $B=I$. Writing out the conditions for the invariance of the cubic $y_{i}$ terms, one obtains $C=0$ for general choice of $F$. Finally, the automorphism has to fix the octic $f_{8}(\vec{x})=0$, so for general $f_{8}$, the only possibility is $A=\delta I$ and then clearly $\delta= \pm 1$. Thus up to constant, $A= \pm I, B=I, C=0$, where the final sign is part of the $\mathbb{C}^{*}$-action in the definition of weighted projective space.

The proof for a general $X_{12} \subset \mathbb{P}^{4}\left[1^{2}, 2^{2}, 6\right]$ is completely analogous, so it is omitted.

\section{The conclusion}

I will need the following rather standard result:

Proposition 4.1 Let $\mathcal{X} \rightarrow B$ be a family of Calabi-Yau threefolds with canonical singularities over a complex space $B$, having a simultaneous resolution $\mathcal{Y} \rightarrow \mathcal{X}$ over $B$. Let $\mathcal{L}$ be a relatively ample relative Cartier divisor on $\mathcal{X}$. Let $\operatorname{Aut}_{B}(\mathcal{X}, \mathcal{L})$ be the scheme of relative automorphisms in the family. Then $\operatorname{Aut}_{B}(\mathcal{X}, \mathcal{L})$ is finite and unramified over $B$.

Proof $\operatorname{Aut}_{B}(\mathcal{X}, \mathcal{L})$ is unramified over $B$, as the fibres of the family $\mathcal{X} \rightarrow B$ are varieties without infinitesimal automorphisms. Quasi-finiteness is clear, and properness follows from the valuative criterion (see e.g. [7, Lemma 4.2]).

Proposition 4.2 Let $X$ be a general member of one of the two families, $Y$ the Calabi-Yau resolution. Let $v \in S \backslash S_{E}$ be a deformation direction as in Section 1, and $\mathcal{Y} \rightarrow \Delta$ the corresponding family with flop $\mathcal{Y}^{+} \rightarrow \Delta$. Assume that for all $t \in U$ in a dense set $U \subset \Delta^{*}$, there exists an isomorphism $Y_{t} \cong Y_{t}^{+}$. Then the variety $X$ has nontrivial automorphism group, respectively automorphism group larger than $\mathbb{Z} / 2 \mathbb{Z}$.

Lemma 4.3 Assume that for some $t \in \Delta^{*}$, there exists an isomorphism $Y_{t} \cong Y_{t}^{+}$. Then $\operatorname{Aut}\left(X_{t}\right)$ is nontrivial, respectively larger than $\mathbb{Z} / 2 \mathbb{Z}$.

Proof Assume that $Y_{t} \cong Y_{t}^{+}$, then the flop corresponds to a nontrivial birational automorphism $\psi \in \operatorname{Birat}\left(Y_{t}\right)$, not the identity on the complement of the exceptional locus (and not the involution $j$ ). So it descends to a nontrivial birational automorphism $\bar{\psi} \in \operatorname{Birat}\left(X_{t}\right)$. On the other hand, using the fact that any isomorphism $Y_{t} \cong Y_{t}^{+}$must identify faces of the nef cones of the same type, it is easy to check that $\bar{\psi}$ must fix a suitable multiple of the ample generator of the Picard group of $X_{t}$. Hence $\bar{\psi}$ is biregular by [12, 2.1.6].

Proof of Proposition 4.2 Let $\mathcal{X} \rightarrow \Delta$ be the contracted family, $\mathcal{N}$ a relatively ample sheaf on $\mathcal{X}$. If there exists an isomorphism $Y_{t} \cong Y_{t}^{+}$for all $t \in U$, there is a nontrivial element in $\operatorname{Aut}_{U}(\mathcal{X}, \mathcal{N})$ for all $t \in U$. The statement now follows from Proposition 4.1.

Let $\mathcal{D}$ denote the period domain parameterizing polarized Hodge structures on the $\mathbb{Z}$-module $H^{3}(Y)$. Let

$$
\Gamma=\operatorname{Aut}\left(H^{3}(Y), Q_{Y}\right)
$$


be the corresponding arithmetic monodromy group. The following is the main result of the paper:

Theorem 4.4 Let $Y$ be a resolution of a general $X_{8} \subset \mathbb{P}^{4}\left[1^{2}, 2^{3}\right]$ or $X_{12} \subset \mathbb{P}^{4}\left[1^{2}, 2^{2}, 6\right]$. Then (weak) global Torelli fails for $Y$ : the period map is finite of degree at least two from the deformation space of $Y$ modulo isomorphisms onto its image in $\mathcal{D} / \Gamma$, the period domain modulo monodromy.

Proof Finiteness of the period map follows from [15, 4.3], keeping in mind Lemma 2.7 above. The rest follows from Theorem 1.1, Proposition 3.1 and Proposition 4.2.

Remark 4.5 Fixing a marking of the cohomology of $Y$ and using the Gauss-Manin connection in the bundle $R^{3} \pi_{*} \mathbb{C}$ over the base of the Kuranishi space $S$, one obtains the (local) period map $\varphi: S \rightarrow \mathcal{D}$. By the Infinitesimal Torelli theorem, $\varphi$ is an embedding. However, this does not contradict the above result: the period map is not invariant under the involution $\alpha$ acting on $S$, it is only equivariant with respect to an element $\gamma \in \Gamma$ fixing the period point of the central fibre $Y$. To conclude the paper, I give a geometric description of $\gamma$.

There is a split exact sequence of Hodge structures

$$
0 \rightarrow H^{3}(X) \rightarrow H^{3}(Y) \rightarrow H^{1}(C)[-1] \rightarrow 0
$$

where the first map is pullback, whereas the second is the dual of the cylinder homomorphism given by the family $E \rightarrow C$ of rational curves in $Y$. Note that the Hodge structure on $H^{3}(X)$ is pure, as $X$ has only quotient singularities.

Proposition 4.6 The element $\gamma \in \Gamma$ is the involution of the Hodge structure $H^{3}(Y)$ that fixes $H^{3}(X)$ and reflects the sub-Hodge structure $H^{1}(C)[-1]$ generated by the family $E \rightarrow C$ of rational curves.

Proof By construction, the image of $H^{3}(X)$ is fixed by the involution $\gamma$. On the other hand, it is clear that $\gamma$ induces the same action as $\alpha$ on the space $H^{2,1}(Y) \cong H^{1}\left(Y, T_{Y}\right)$, the latter isomorphism being well-defined up to a constant. Thus the corank of the submodule fixed by $\gamma$ is at least $2 g$ by Lemma 1.5. Hence the fixed submodule is exactly $H^{3}(X)$ and this concludes the proof.

\section{References}

[1] P. Aspinwall and D. Morrison, Chiral rings do not suffice: $N=(2,2)$ theories with nonzero fundamental group, Phys. Lett. B 334 (1994) 79-86.

[2] C. Borcea, On desingularized Horrocks-Mumford quintics, J. reine angew. Math. 421 (1991), 23-41.

[3] P. Candelas, P.S. Green and T. Hübsch, Rolling among Calabi-Yau vacua, in: Superstrings '89 (Trieste, 1989, eds. M. Green et al), 366-421, World Sci. Publishing, River Edge, NJ, 1990.

[4] P. Candelas, X. de la Ossa, A. Font, S. Katz and D. Morrison, Mirror symmetry for two-parameter models I, Nucl. Phys. B 416 (1994) 481-538.

[5] D.A. Cox, The homogeneous coordinate ring of a toric variety, J. Alg. Geom. 4 (1995) 17-50. 
[6] O. Debarre, Un contre-exemple au théorème de Torelli pour les variétés symplectiques irréductibles, C. R. Acad. Sci. Paris Sér. I Math. 299 (1984), 681-684.

[7] B. Fantechi and R. Pardini, Automorphisms and moduli spaces of varieties with ample canonical class via deformations of abelian covers, Comm. Algebra 25 (1997) 1413-1441.

[8] T. Fujita, On hyperelliptic polarized varieties, Tohoku Math. J. (2) 35 (1983), 1-44.

[9] D. Huybrechts, Compact hyperkähler manifolds: basic results, Inv. Math. 135 (1999), 63-113.

[10] S. Katz, D.R. Morrison and M.R. Plesser, Enhanced gauge symmetry in type II string theory, Nucl. Phys. B 477 (1996) 105-140.

[11] J. Kollár, Flops, Nagoya Math. J. 113 (1989), 15-36.

[12] J. Kollár, Flips, flops, minimal models, etc, Surveys in differential geometry 113-199, Lehigh Univ., Bethlehem, PA, 1991.

[13] J. Kollár and S. Mori, Classification of three-dimensional flips, J. Amer. Math. Soc. 5 (1992) 533703.

[14] Z. Ran, Deformations of maps, in: Algebraic curves and projective geometry (Trento, 1988, eds. E. Ballico and C. Ciliberto), 246-253, LNM 1389, Springer, Berlin, 1989.

[15] B. Szendrői, Some finiteness results for Calabi-Yau threefolds, Preprint alg-geom/9708011, to appear in J. Lon. Math. Soc.

[16] B. Szendröi, Calabi-Yau threefolds and the Torelli problem, PhD Thesis, University of Cambridge, in preparation.

[17] G. Tian, Smoothness of the universal deformation space of compact Calabi-Yau manifolds and its Peterson-Weil metric, in: Mathematical aspects of string theory (ed. S.-T. Yau) 629-646, World Scientific, Singapore, 1987.

[18] A. Todorov, The Weil-Petersson geometry of the moduli space of $\mathrm{SU}(n \geq 3)$ (Calabi-Yau) manifolds. I. Comm. Math. Phys. 126 (1989), 325-346.

[19] C. Voisin, A generic Torelli theorem for the quintic threefold, in: New Trends in Algebraic Geometry (Warwick, 1996, eds. K. Hulek et al) Cambridge University Press, Cambridge 1999.

[20] P.M.H. Wilson, The Kähler cone on Calabi-Yau threefolds, Inv. Math. 107 (1992) 561-583, 114 (1993), 231-233.

[21] P.M.H.Wilson, Symplectic deformations of Calabi-Yau threefolds, J. Diff. Geom. 45 (1997), 611-637.

[22] P.M.H. Wilson, Flops, Type III contractions and Gromov-Witten invariants on Calabi-Yau threefolds, in: New Trends in Algebraic Geometry (Warwick, 1996, eds. K. Hulek et al) Cambridge University Press, Cambridge 1999.

Department of Pure Mathematics and Mathematical Statistics

UNIVERSity OF CAMBRIDGE

16 Mill Lane, Cambridge, CB2 1SB, UK

FAX: +441223337920

E-MAIL ADDRESS: balazs@dpmms.cam.ac.uk 